\title{
Long Time Behavior of Entire Solutions to Bistable Reaction Diffusion Equations
}

\author{
Yang Wang* and Xiong Li
}

\begin{abstract}
The first aim in the paper is to prove the local exponential asymptotic stability of some entire solutions to bistable reaction diffusion equations via the supersub solution method. If the integral of the reaction term $f$ over the interval $[0,1]$ is positive, we not only obtain the similar asymptotic stability result found by Yagisita in 2003, but also simplify the proof. The asymptotic stability result for the case $\int_{0}^{1} f(u) d u<0$ is also obtained, which is not considered by Yagisita. After that, the asymptotic behavior of entire solutions as $t \rightarrow+\infty$ is investigated, since the other side was completely known. Here, the result is established by use of the asymptotic stability of constant solutions and pairs of diverging traveling front solutions, instead of constructing the super-sub solutions as usual. Finally, for the special bistable case $f(u)=u(1-u)(u-\alpha), \alpha \in(0,1)$, we prove the entire solution continuously depends on $\alpha$.
\end{abstract}

\section{Introduction}

In this paper we focus on the following reaction diffusion equation

$$
\partial_{t} u=\partial_{x x} u+f(u), \quad x \in \mathbb{R}
$$

where $f \in C^{2}(\mathbb{R}), f(0)=f(\alpha)=f(1)=0, \alpha$ is the unique zero point of $f$ in the interval $(0,1)$, and $f^{\prime}(0), f^{\prime}(1)<0$.

In this case, (1.1) is called a bistable equation and the background can be found in [2, 5,21$]$ and references therein. This model can illustrate that a nerve has been treated with certain toxins as stated in [5]. It can also be used to describe a bistable active transmission line introduced in 21. For more general reaction terms, Aronson and Weinberger in [1] used it to describe the heterozygote inferiority case and also pointed out that some flame propagation problems in chemical reactor theory can be demonstrated by equations of the

Received November 16, 2019; Accepted May 5, 2020.

Communicated by Jann-Long Chern.

2010 Mathematics Subject Classification. 35K57, 35B35, 35B41.

Key words and phrases. entire solutions, stability, reaction diffusion equations.

Wang was partially supported by the NSFC (11901366) and the Natural Science Foundation of Shanxi Province (201801D221008).

Li was partially supported by the NSFC (11971059).

*Corresponding author. 
form (1.1). If the reaction term $f$ has no any zeros in the interval $(0,1)$, also in [1], they used it to describe the heterozygote intermediate case and at this time, (1.1) becomes the famous KPP-Fisher equation, which is monostable and had been studied in [10,17.

In recent years, the existence, uniqueness, stability and other properties of traveling wave solutions of (1.1) have been investigated extensively, for example, see [1,2,6 10, 17].

A function $\phi(\xi), \xi=x+c t$, is called a traveling wave solution of (1.1) connecting 0 and 1 with the wave speed $c$, if it satisfies

$$
\phi^{\prime \prime}(\xi)-c \phi^{\prime}(\xi)+f(\phi(\xi))=0, \quad \lim _{\xi \rightarrow-\infty} \phi(\xi)=0, \quad \lim _{\xi \rightarrow+\infty} \phi(\xi)=1
$$

which is actually monotone increasing proved in [8,22]. Moreover, the monotone traveling wave solution is also known as the traveling front solution.

However, it is not enough to understand the dynamical structure of (1.1) by only considering traveling wave solutions. Recently, the existence of entire solutions, which are classical solutions and defined for all $(x, t) \in \mathbb{R} \times \mathbb{R}$, is widely discussed. From the dynamical view, the study of entire solutions is essential for a full understanding of the transient dynamics and the structures of the global attractor as mentioned in [19,20].

In [14], for the monostable reaction diffusion equation, Hamel and Nadirashvili proved the existence of entire solutions by the comparison theorem, supersolution and subsolution, which consists of traveling front solutions and solutions to the diffusion-free equation. Meanwhile, they also pointed out that the solutions to (1.1) depending only on $t$ and traveling wave solutions are typical examples of entire solutions and showed various entire solutions of 1.1 in their subsequent paper 15. If $\int_{0}^{1} f(u) d u>0$, which implies that wave speeds of any traveling front solutions of (1.1) must be positive, Yagisita in 28 revealed that the annihilation process is approximated by a backward global solution of (1.1), which is in fact an entire solution.

For Allen-Cahn equation

$$
\partial_{t} u=\partial_{x x} u+u(1-u)(u-\alpha), \quad \alpha \in(0,1),
$$

which is a special example of (1.1), Fukao, Morita and Ninomiya in 11] proposed a simple proof for the existence of entire solutions, which was already found in [28] by using the super-sub solution method and the exact traveling front solutions. Moreover, Guo and Morita in 13 extended the conclusions in 14, 28 to more general case. If $\int_{0}^{1} f(u) d u<0$, which implies that wave speeds $c$ of any traveling front solutions of (1.1) must be negative, Chen and Guo in 3 used the quite different method to construct the super-sub solutions to obtain the existence and uniqueness of entire solutions to (1.1), which are different from those in [11, 13, 28]. Very recently, Matano and Poláčik in [19] proved that there is an entire solution connecting the origin to the solution only depending on $x$ of (1.1), and 
Chen et al. in [4] proved that there are no entire solutions originating from more than four traveling front solutions.

In this paper we will firstly investigate the asymptotic behavior of entire solutions to (1.1) as $t \rightarrow+\infty$, since the exact asymptotic behavior as $t \rightarrow-\infty$ was found in [3, 13]. We conjecture that the long time behavior of entire solutions to $[1.1$ found in 3,13 may be controlled by some asymptotic stable states of (1.1) defined in 6]. Luckily, Fife in 6 pointed out several kinds of asymptotic stable states, including constant solutions $u \equiv 0$, $u \equiv 1$, traveling wave solutions, diverging pairs of traveling wave solutions. With these results, the long time behavior of these entire solutions can be established.

Secondly, Yagisita in 28 proved that the entire solution to 1.1 is local exponential asymptotic stable by the asymptotic stability of the constructed invariant manifold. Also the local Lyapunov stability of entire solutions to (1.1) with nonlocal delays was obtained in 26 by the super-sub solution method. By means of establishing the different super-sub solutions of 21.1] from that in [26], we further prove the local exponential asymptotic stability of the entire solutions to (1.1) found in [3, 13]. Eventually, we will prove the entire solution to (1.3) continuously depend on $\alpha$ followed the method offered by [14].

The rest is organized as follows. For the reader's convenience, in Section 2, we introduce some known results about the asymptotic stability of constant solutions, traveling wave solutions and diverging pairs of traveling wave solutions to 1.1. Then, in Section 3 . we investigate the long time behavior of entire solutions to $\sqrt{1.1}$ and prove the local exponential asymptotic stability of entire solutions by the super-sub solution method. In the end, in Section 4, we prove the continuity of the entire solution to $(1.3)$ in $\alpha$.

\section{Some known results}

In the sequel, we will investigate the long time behavior of entire solutions to 1.1 in the Banach space

$$
U C(\mathbb{R}):=\{u \in C(\mathbb{R}): u \text { is bounded and uniformly continuous in } \mathbb{R}\}
$$

with the norm $\|u\|:=\sup _{x \in \mathbb{R}}|u(x)|$. For this purpose, we present the initial condition

$$
u(x, 0)=u_{0}(x), \quad x \in \mathbb{R}
$$

where $u_{0}(x) \in U C(\mathbb{R})$, and let the function $u\left(x, t ; u_{0}\right)$ be the solution of equation (1.1) with the initial condition (2.1). Now we introduce an interior Schauder estimate in the following lemma without the proof.

Lemma 2.1. Suppose that $u\left(x, t ; u_{0}\right)$ is a bounded solution to (1.1) with 2.1) for $(x, t) \in$ $\mathbb{R} \times[0,+\infty)$, and $\left\|u\left(\cdot, t ; u_{0}\right)\right\| \leq M_{0}$ for some constant $M_{0}>0$ and all $t \geq 0$. Assume 
that the reaction term $f \in C^{2}(\mathbb{R})$, and there exists a positive constant $M_{1}$ such that $\|f\|,\left\|f^{\prime}\right\|$ and $\left\|f^{\prime \prime}\right\| \leq M_{1}$ on $\left[-M_{0}, M_{0}\right]$. Then there is a positive constant $M_{2}$ such that $\left\|\partial_{t} u\left(\cdot, t ; u_{0}\right)\right\|,\left\|\partial_{x} u\left(\cdot, t ; u_{0}\right)\right\|,\left\|\partial_{x x} u\left(\cdot, t ; u_{0}\right)\right\| \leq M_{2}$ for all $t \in[1,+\infty)$, where $M_{2}$ depends on $M_{0}$ and $M_{1}$ only.

Here and thereafter, $M$ stands for general positive constant and $M_{i}, i=1,2, \ldots$, stand for special positive constants.

In this paper we mainly are concerned with the local exponential asymptotic stability of entire solutions to (1.1). Now we introduce the definition of the local exponential asymptotic stability.

Definition 2.2. Suppose $v(x, t)$ is an entire solution to (1.1). If there are $\delta>0, M>0$, $a>0$ and $t_{0}>0$ such that when $\left\|u_{0}(\cdot)-v(\cdot, 0)\right\| \leq \delta$,

$$
\left\|u\left(\cdot, t ; u_{0}\right)-v(\cdot, t)\right\| \leq M e^{-a t}, \quad \forall t \geq t_{0}
$$

then $v(x, t)$ is called local exponential asymptotic stable.

Now we list the known results about the stability of constant solutions, traveling wave solutions and diverging pairs of traveling wave solutions (constructed by traveling wave solutions and their reflects), which will be used later.

First of all, we state the asymptotic stability of constant solutions $u \equiv 0$ and $u \equiv 1$, which had been proved in $[7$.

Lemma 2.3. Suppose that $0 \leq u_{0} \leq 1$ is a continuous function. If $\int_{0}^{1} f(u) d u \geq 0$ and $\liminf _{x \rightarrow \pm \infty} u_{0}(x)>\alpha$, then

$$
\lim _{t \rightarrow+\infty}\left\|u\left(\cdot, t ; u_{0}\right)-1\right\|=0
$$

if $\int_{0}^{1} f(u) d u \leq 0$ and $\lim \sup _{x \rightarrow \pm \infty} u_{0}(x)<\alpha$, then

$$
\lim _{t \rightarrow+\infty}\left\|u\left(\cdot, t ; u_{0}\right)\right\|=0 .
$$

Moreover if $\inf _{x \in \mathbb{R}} u_{0}(x)>\alpha$, then $\lim _{t \rightarrow+\infty}\left\|u\left(\cdot, t ; u_{0}\right)-1\right\|=0 ;$ if $\sup _{x \in \mathbb{R}} u_{0}(x)<\alpha$, then $\lim _{t \rightarrow+\infty}\left\|u\left(\cdot, t ; u_{0}\right)\right\|=0$.

The global exponential asymptotic stability of traveling wave solutions of (1.1) had been proved in [8].

Lemma 2.4. Suppose that $\phi$ is the solution to 1.2 and $0 \leq u_{0} \leq 1$ is a continuous function. If $\limsup _{x \rightarrow-\infty} u_{0}(x)<\alpha$, $\liminf _{x \rightarrow+\infty} u_{0}(x)>\alpha$, then there are some constants $x_{0}, M_{3}>0$ and $\omega_{1}>0$ such that

$$
\left|u\left(x, t ; u_{0}\right)-\phi\left(x+c t-x_{0}\right)\right|<M_{3} e^{-\omega_{1} t}, \quad t \geq 0, x \in \mathbb{R}
$$


if $\lim \sup _{x \rightarrow+\infty} u_{0}(x)<\alpha, \liminf _{x \rightarrow-\infty} u_{0}(x)>\alpha$, then there are some constants $x_{1}$, $M_{4}>0$ and $\omega_{2}>0$ such that

$$
\left|u\left(x, t ; u_{0}\right)-\phi\left(-x+c t-x_{1}\right)\right|<M_{4} e^{-\omega_{2} t}, \quad t \geq 0, x \in \mathbb{R} .
$$

In addition, the local asymptotic stability of traveling wave solutions of 1.1 had been proved in 22, 23] by the different ways compared with the method in [8]. The stability of traveling wave solutions in $\mathbb{R}^{n}$ can be found in [18, 25, 27]. For more general reaction terms, the authors in the papers [12,24] proved the stability of traveling wave solutions.

As stated in [6], there are four kinds of bounded stationary solutions of equation (1.1), which are solutions of

$$
\frac{d^{2} u}{d x^{2}}+f(u)=0
$$

that is, the solution with a single minimum point, the solution with a single maximum point, the periodic solution and the monotone solution. In fact, the solution with a single maximum point or a single minimum point is the homoclinic orbit. More importantly, the author also proved that the solution of $(2.2)$ with a maximum or minimum at a finite value of $x$ are unstable, which means that only the monotone solution, namely the heteroclinic orbit, is stable. From the views of parabolic equations, the monotone solution of $(2.2)$ is the solution to 1.2 with $c=0$ and the stability had been proved in 8 . Specially, for (1.3) with $\alpha \neq 1 / 2$, the author in [16] pointed out that $\phi(\xi)$ is the solution of $(2.2)$ with $\phi(+\infty)=\phi(-\infty)$ if and only if $c=0$. However, in the classical two species Lotka-Volterra competition model, there exists a similar homoclinic orbit with $c \neq 0$.

Finally, when $\int_{0}^{1} f(u) d u \neq 0$, the authors in 8 discussed the asymptotic stability of diverging pairs of traveling wave solutions, where the initial function $u_{0}(x)$ is assumed to lie above the line $u=\alpha$ for $x$ in the large symmetrical interval about the origin and $\lim \sup _{x \rightarrow \pm \infty} u_{0}(x)<\alpha$, or lie below the line $u=\alpha$ for $x$ in the large symmetrical interval about the origin and $\liminf _{x \rightarrow \pm \infty} u_{0}(x)>\alpha$, that is, there are two pairs of positive constants $\widehat{\beta}_{1}, \check{\beta}_{1}, L_{1}, L_{2}$ such that $u_{0}(x)>\alpha+\widehat{\beta}_{1}$ for $|x|<L_{1}$, or $u_{0}(x)<\alpha-\check{\beta}_{1}$ for $|x|<L_{2}$, where the large constants $L_{1}, L_{2}$ depend on $\widehat{\beta}_{1}, \check{\beta}_{1}$ and $f$. However, the explicit expressions of $L_{1}, L_{2}$ are not given in [8]. Now, according to the proof of Lemma 6.1 in [8], the lower bounds of $L_{1}, L_{2}$ can be obtained, respectively.

In what follows, the estimates of the two lower bounds are given. It is easy to see that the characteristic equations of the linearized equations of 1.2 at $u=0$ and $u=1$ are

$$
\lambda^{2}-c \lambda+f^{\prime}(0)=0, \quad \mu^{2}-c \mu+f^{\prime}(1)=0 .
$$

The corresponding eigenvalues are

$$
\lambda_{1}=\frac{c+\sqrt{c^{2}-4 f^{\prime}(0)}}{2}, \quad \lambda_{2}=\frac{c-\sqrt{c^{2}-4 f^{\prime}(0)}}{2},
$$




$$
\mu_{1}=\frac{c+\sqrt{c^{2}-4 f^{\prime}(1)}}{2}, \quad \mu_{2}=\frac{c-\sqrt{c^{2}-4 f^{\prime}(1)}}{2} .
$$

Therefore, there are some positive constants $M_{5}, M_{6}, M_{7}$ and $M_{8}$ such that

$$
\begin{array}{ll}
M_{5} e^{\mu_{2} \xi} \leq 1-\phi(\xi) \leq M_{6} e^{\mu_{2} \xi}, & \xi \geq 0, \\
M_{7} e^{\lambda_{1} \xi} \leq \phi(\xi) \leq M_{8} e^{\lambda_{1} \xi}, & \xi \leq 0,
\end{array}
$$

which are also given in [13].

Since $f^{\prime}(\alpha)>0$, then

$$
w:=\max _{u \in[0,1]} f^{\prime}(u)>0 .
$$

Also since $\lim _{u \rightarrow 1^{-}} \frac{f(u)}{1-u}=-f^{\prime}(1)$ and $\lim _{u \rightarrow 0^{+}} \frac{f(u)}{u}=f^{\prime}(0)$, then the functions $\frac{f(u)}{1-u}$ and $\frac{f(u)}{u}$ are continuous in the interval $[0,1]$ if we define the function $\frac{f(u)}{1-u}$ as $-f^{\prime}(1)$ at $u=1$, $\frac{f(u)}{u}$ as $f^{\prime}(0)$ at $u=0$. Thus there is a positive constant $M_{9}$ such that

$$
|f(u)| \leq M_{9}(1-u), \quad|f(u)| \leq M_{9} u, \quad u \in[0,1] .
$$

Give any $\widehat{\beta}_{1}>0$, choose $\widehat{q}_{0}, \widehat{q}_{1}$ as $0<1-\widehat{q}_{1}<1-\widehat{q}_{0}<\alpha+\widehat{\beta}_{1}$, let $\widehat{\mu}_{1}>0, \widehat{\beta}$ and $\widehat{M}$ be corresponding to $\mu_{1}, \beta$ and $M$ in the proof of Lemma 6.1 in $\left[8\right.$ respectively, choose $\widehat{\mu}_{2}$, $\widehat{M}^{\prime}, \widehat{\varphi}_{0}$ as

$$
\begin{aligned}
& 0<\widehat{\mu}_{2}<\min \left\{-\mu_{2} c, \widehat{\mu}_{1}\right\}, \quad \widehat{M}^{\prime}=\frac{w+M_{9}}{c \widehat{\beta} \mu_{2}} M_{6}-\frac{w+\widehat{\mu}_{2}}{\widehat{\beta} \widehat{\mu}_{2}} \widehat{q}_{0}<0, \\
& \widehat{\varphi}_{0}<\min \left\{\widehat{M}^{\prime}, \widehat{M}^{\prime}-\frac{1}{\mu_{2}} \ln \frac{\widehat{q}_{1}-\widehat{q}_{0}}{M_{6}}, \widehat{M}^{\prime}-\frac{1}{\mu_{2}} \ln \frac{\left(\widehat{\mu}_{1}-\widehat{\mu}_{2}\right) \widehat{q}_{0}}{M_{6} M_{9}}\right\},
\end{aligned}
$$

then a lower bound of $L_{1}$ can be obtained as below

$$
L_{1} \geq \widehat{M} \geq \max \left\{-\widehat{\varphi}_{0},-\frac{1}{\lambda_{1}} \ln \frac{1-\alpha-\widehat{\beta}_{1}}{M_{8}}-\widehat{\varphi}_{0}\right\} .
$$

Similarly, give any $0<\check{\beta}_{1}<\alpha$, choose $\check{q}_{0}, \check{q}_{1}$ as $0<\alpha-\check{\beta}_{1}<\check{q}_{0}<\check{q}_{1}<\alpha$, let $\check{\mu}_{1}>0, \check{M}$ and $\check{\beta}$ be corresponding to $\mu_{1}^{\prime}, M^{\prime}$ and $\beta^{\prime}$ in the proof of Lemma 6.1 in 8 respectively, choose $\check{\mu}_{2}, \check{M}^{\prime}, \check{\varphi}_{0}$ as

$$
\begin{aligned}
& 0<\check{\mu}_{2}<\min \left\{-\lambda_{1} c, \check{\mu}_{1}\right\}, \quad \check{M}^{\prime}=\frac{w+M_{9}}{c \lambda_{1} \check{\beta}} M_{8}-\frac{w+\check{\mu}_{2}}{\check{\beta}_{\tilde{\mu}}} \check{q}_{0}<0, \\
& \check{\varphi}_{0}<\min \left\{\check{M}^{\prime}, \check{M}^{\prime}+\frac{1}{\lambda_{1}} \ln \frac{\check{q}_{1}-\check{q}_{0}}{M_{8}}, \check{M}^{\prime}+\frac{1}{\lambda_{1}} \ln \frac{\left(\check{\mu}_{1}-\check{\mu}_{2}\right) \check{q}_{0}}{M_{8} M_{9}}\right\},
\end{aligned}
$$

then a lower bound of $L_{2}$ is given by

$$
L_{2} \geq \check{M} \geq \max \left\{-\check{\varphi}_{0}, \frac{1}{\mu_{2}} \ln \frac{\alpha-\check{\beta}_{1}}{M_{6}}-\check{\varphi}_{0}\right\} .
$$

Therefore we can refine Theorem 3.2 in $[8]$ and obtain the following lemma. 
Lemma 2.5. Suppose that $\phi$ is the solution to (1.2), and $0 \leq u_{0} \leq 1$ is a continuous function. If $\int_{0}^{1} f(s) d s>0$, $\limsup _{x \rightarrow \pm \infty} u_{0}(x)<\alpha$ and $u_{0}(x)>\alpha+\widehat{\beta}_{1}$ for $|x|<L_{1}$, then there are some constants $x_{2}, x_{3}$ and positive constants $M_{10}, \omega_{3}$ such that

$$
\begin{aligned}
\left|u\left(x, t ; u_{0}\right)-\phi\left(x+c t-x_{2}\right)\right|<M_{10} e^{-\omega_{3} t}, & x<0, t \geq 0 \\
\left|u\left(x, t ; u_{0}\right)-\phi\left(-x+c t-x_{3}\right)\right|<M_{10} e^{-\omega_{3} t}, & x>0, t \geq 0 .
\end{aligned}
$$

If $\int_{0}^{1} f(s) d s<0, \liminf _{x \rightarrow \pm \infty} u_{0}(x)>\alpha$ and $u_{0}(x)<\alpha-\check{\beta}_{1}$ for $|x|<L_{2}$, then there are some constants $x_{4}, x_{5}$ and positive constants $M_{11}, \omega_{4}$ such that

$$
\begin{aligned}
\left|u\left(x, t ; u_{0}\right)-\phi\left(x+c t-x_{4}\right)\right|<M_{11} e^{-\omega_{4} t}, & x>0, t \geq 0 \\
\left|u\left(x, t ; u_{0}\right)-\phi\left(-x+c t-x_{5}\right)\right|<M_{11} e^{-\omega_{4} t}, & x<0, t \geq 0 .
\end{aligned}
$$

More interestingly, the author in [6] conjectured that when the bounded initial function $u_{0}$ is away from $\alpha$ for large $|x|$, the asymptotic stable solution may only be one of the four kinds: $u \equiv 0, u \equiv 1$, the traveling wave solution, the diverging pairs of traveling wave solutions, while other solutions are unstable. Although the conjecture is partially solved in the paper [7], it has been not completely solved and is still open. Moreover, we also remark that $(1.1)$ admits a traveling wave solution $\widehat{\phi}$ connecting $\alpha$ and 1 , and there are some results about $u\left(x, t ; u_{0}\right)$ converging to $\widehat{\phi}$ when $\alpha \leq u_{0} \leq 1$, referring to 17 . The authors in 20 also presented that if $u_{0}(x)<\alpha$ for $x$ in some interval of $x$-axis, then whether $u\left(x, t ; u_{0}\right)$ converges to $\widehat{\phi}$ or not? For example, if $u_{0}$ satisfies the following condition

$$
\lim _{x \rightarrow+\infty} u_{0}(x)=1, \quad \lim _{x \rightarrow-\infty} u_{0}(x)=\alpha,
$$

and there exists $\widetilde{x}$ such that when $x \leq \widetilde{x}, u_{0}(x)<\alpha$,

then whether $u\left(x, t ; u_{0}\right)$ converges to $\widehat{\phi}$ or not? Thus this open problem contains the opposite side of the above conjecture. That is, suppose that the initial condition $u_{0}(x)$ is bounded and, when $|x|$ is sufficiently large, $u_{0}(x)$ is not far away from $\alpha$ at least on one side of $x$-axis, then wether $u\left(x, t ; u_{0}\right)$ converges to $\widehat{\phi}$ or not? In a word, the proof of this open problem will enforce the understanding of the conjecture.

3. The local exponential asymptotic stability of entire solutions to the bistable equation

In this section, we will discuss the local exponential asymptotic stability of entire solutions to (1.1) found in 3, 13 respectively, and their asymptotic behaviors when $t$ converges to $+\infty$. 
Before stating the main results, we do some preparations. Firstly, in order to prove the existence of entire solutions, the authors in [13] constructed two pairs of different supersub solutions corresponding to $f^{\prime}(0) \leq f^{\prime}(1)$ and $f^{\prime}(0)>f^{\prime}(1)$, respectively. Moreover, it follows from 8 that

$$
\text { if } \int_{0}^{1} f(u) d u \gtreqless 0, \quad \text { then } c \gtreqless 0 .
$$

Hence we will discuss the long time behaviors and stabilities of entire solutions under the following four cases:

(C1) $\int_{0}^{1} f(u) d u>0$ and $f^{\prime}(0)>f^{\prime}(1)$

(C2) $\int_{0}^{1} f(u) d u>0$ and $f^{\prime}(0) \leq f^{\prime}(1)$;

(C3) $\int_{0}^{1} f(u) d u<0$ and $f^{\prime}(0)>f^{\prime}(1)$

(C4) $\int_{0}^{1} f(u) d u<0$ and $f^{\prime}(0) \leq f^{\prime}(1)$.

Secondly, for the sake of the proof of the uniqueness of entire solutions, similar to [3], we introduce the metable dynamics of (1.1). We call the solution $u(x, t)$ of 1.1 satisfying the condition $\mathbb{M}^{+}$, if there exist constants $d_{1}>0$ and $T_{1} \in \mathbb{R}$, functions $l_{1}(t)$ and $m_{1}(t)$ such that for all $t \leq T_{1}$,

$$
u(x, t) \begin{cases}\leq \alpha_{1}, & \forall x \in\left[\min \left\{l_{1}(t)+d_{1}, m_{1}(t)-d_{1}\right\}, \max \left\{l_{1}(t)+d_{1}, m_{1}(t)-d_{1}\right\}\right] \\ \geq \alpha_{2}, & \forall x \in\left(-\infty, l_{1}(t)\right] \cup\left[m_{1}(t),+\infty\right)\end{cases}
$$

where $\alpha_{1}$ and $\alpha_{2}$ are some constants satisfying $f \neq 0$ in $\left(0, \alpha_{1}\right] \cup\left[\alpha_{2}, 1\right)$. Similarly, the solution $u(x, t)$ of $(1.1)$ is called to satisfy the condition $\mathbb{M}^{-}$, if there exist constants $d_{2}>0$ and $T_{2} \in \mathbb{R}$, functions $l_{2}(t)$ and $m_{2}(t)$ such that for all $t \leq T_{2}$,

$$
u(x, t) \begin{cases}\leq \alpha_{1}, & \forall x \in\left(-\infty, l_{2}(t)\right] \cup\left[m_{2}(t),+\infty\right], \\ \geq \alpha_{2}, & \forall x \in\left[\min \left\{l_{2}(t)+d_{2}, m_{2}(t)-d_{2}\right\}, \max \left\{l_{2}(t)+d_{2}, m_{2}(t)-d_{2}\right\}\right]\end{cases}
$$

where $\alpha_{1}$ and $\alpha_{2}$ are some constants satisfying $f \neq 0$ in $\left(0, \alpha_{1}\right] \cup\left[\alpha_{2}, 1\right)$.

We initially discuss entire solutions found in Theorem 1.1 from $[13$. By the method of the proof of the uniqueness in $[3]$ and Lemma 2.3 , we can prove the uniqueness of entire solutions and obtain the following result.

Theorem 3.1. Suppose that $\int_{0}^{1} f(u) d u>0$. Let $\phi$ be the solution to 1.2 with the wave speed $c$. Then for any given constants $y_{1}$ and $y_{2}$, there is a unique entire solution (up to a translation in $t$ and $x) u_{1}(x, t)$ of (1.1) defined for all $(x, t) \in \mathbb{R} \times \mathbb{R}$ such that 
$0<u_{1}(x, t)<1, \partial_{t} u_{1}(x, t)>0$ and

$$
\begin{gathered}
\lim _{t \rightarrow-\infty}\left\{\sup _{x \geq 0}\left|u_{1}(x, t)-\phi\left(x+c t+y_{1}\right)\right|+\sup _{x \leq 0}\left|u_{1}(x, t)-\phi\left(-x+c t+y_{2}\right)\right|\right\}=0, \\
\lim _{t \rightarrow+\infty} \sup _{x \in \mathbb{R}}\left|u_{1}(x, t)-1\right|=0 .
\end{gathered}
$$

Proof. We first consider the case (C1). When $t \leq 0$, it follows from [13] that the supersolution and subsolution of $(1.2)$ are

$$
\begin{aligned}
& \bar{u}_{1}(x, t)=\min \left\{\phi\left(x+p_{1}(t)\right)+\phi\left(-x+p_{1}(t)\right), 1\right\}, \\
& \underline{u}_{1}(x, t)=\max \left\{\phi\left(x+c t+x_{6}\right), \phi\left(-x+c t+x_{6}\right)\right\},
\end{aligned}
$$

where the function $p_{1}(t)(t \leq 0)$ is the solution to

$$
p_{1}^{\prime}(t)=c+M_{12} e^{\lambda_{1} p_{1}(t)}, \quad t<0, \quad p_{1}(0) \leq 0,
$$

$c$ is the wave speed, $\lambda_{1}=\frac{c+\sqrt{c^{2}-4 f^{\prime}(0)}}{2}$, and the expression of $M_{12}>0$ is too long, which can be found in [13], and $x_{6}=p_{1}(0)-\frac{1}{\lambda_{1}} \ln \left(1+\frac{M_{12}}{c}\right)$.

Substituting $t=0$ into the expression of the subsolution $\underline{u}_{1}(x, t)$, we have

$$
\liminf _{x \rightarrow \pm \infty} u_{1}(x, 0) \geq \liminf _{x \rightarrow \pm \infty} \underline{u}_{1}(x, 0)=\lim _{x \rightarrow \pm \infty} \max \left\{\phi\left(x+x_{6}\right), \phi\left(-x+x_{6}\right)\right\}=1>\alpha .
$$

Since $\int_{0}^{1} f(s) d s>0$, it follows from Lemma 2.3 that

$$
\lim _{t \rightarrow+\infty}\left\|u_{1}(\cdot, t)-1\right\|=0
$$

which is the asymptotic behavior of the entire solution $u_{1}(x, t)$ when $t$ converges to $+\infty$. On the other hand, as $t \rightarrow-\infty$, the asymptotic behavior had been already proved in [13].

Since $\underline{u}_{1}\left(x, t+t_{1}\right)<\bar{u}_{1}(x, t)$ for arbitrary $t_{1}$, then the method in [13] to prove the uniqueness of entire solutions is no longer valid. Here we adopt the method in [3] to prove the uniqueness, and only need to verify that $u_{1}(x, t)$ satisfies the condition $\mathbb{M}^{+}$.

By the assumption on $f$, there are some constants $\alpha_{1}<\alpha<\alpha_{2}$ such that $f \neq 0$ in $\left(0, \alpha_{1}\right] \cup\left[\alpha_{2}, 1\right)$. Then from the monotonicity of $\phi$, there exists a positive constant $\widetilde{x}$ such that $\phi\left(\widetilde{x}+x_{6}\right) \geq \alpha_{2}$. Set

$$
l_{1}(t)=c t-\widetilde{x}, \quad m_{1}(t)=-c t+\widetilde{x} .
$$

For any $t \leq 0$, obviously $m_{1}(t) \geq 0 \geq l_{1}(t)$. On one hand, since

$$
u_{1}(x, t) \geq \max \left\{\phi\left(x+c t+x_{6}\right), \phi\left(-x+c t+x_{6}\right)\right\},
$$


then $u_{1}(x, t) \geq \alpha_{2}$ for any $x \in\left(-\infty, l_{1}(t)\right] \cup\left[m_{1}(t),+\infty\right)$. On the other hand, according to 3.1 , for any $\varepsilon \in(0, \alpha)$, there exists a $T_{11}<0$ such that for any $t \leq T_{11}$,

$$
\sup _{x \geq 0}\left|u_{1}(x, t)-\phi\left(x+c t+y_{1}\right)\right| \leq \varepsilon, \quad \sup _{x \leq 0}\left|u_{1}(x, t)-\phi\left(-x+c t+y_{2}\right)\right| \leq \varepsilon .
$$

Then take $d_{1}$ sufficiently large such that

$$
\max \left\{\phi\left(\widetilde{x}-d_{1}+y_{1}\right), \phi\left(\widetilde{x}-d_{1}+y_{2}\right)\right\}<\alpha_{1}-\varepsilon
$$

It is clear that there is a $T_{12}<0$ such that $l_{1}(t)+d_{1} \leq 0 \leq m_{1}(t)-d_{1}$ for any $t \leq T_{12}$. Choose $T_{1} \leq \min \left\{T_{11}, T_{12}\right\}$. Then, for $t \leq T_{1}$ and $0 \leq x \leq m_{1}(t)-d_{1}$,

$$
u_{1}(x, t) \leq \phi\left(m_{1}(t)-d_{1}+c t+y_{1}\right)+\varepsilon=\phi\left(\widetilde{x}-d_{1}+y_{1}\right)+\varepsilon \leq \alpha_{1},
$$

and for $t \leq T_{1}$ and $l_{1}(t)+d_{1} \leq x \leq 0$,

$$
u_{1}(x, t) \leq \phi\left(-l_{1}(t)-d_{1}+c t+y_{2}\right)+\varepsilon=\phi\left(\widetilde{x}-d_{1}+y_{2}\right)+\varepsilon \leq \alpha_{1} .
$$

Thus, $u_{1}(x, t) \leq \alpha_{1}$ for all $t \leq T_{1}$ and $x \in\left[l_{1}(t)+d_{1}, m_{1}(t)-d_{1}\right]$. Therefore, the entire solution $u_{1}(x, t)$ satisfies the condition $\mathbb{M}^{+}$, and from the proofs in the papers [3, 26], it is unique up to a space-time translation.

Finally, we will prove the monotonicity of $u_{1}(x, t)$ with respect to $t$ via the method in 14. From the proof of the existence of entire solutions in the papers [3, 13] and so on, the authors chose the function $u\left(x, t+n ; u_{n}(x,-n)\right):=u_{n}(x, t)$ as the unique classical solution to the following initial problem

$$
\begin{cases}\partial_{t} u_{n}=\partial_{x x} u_{n}+f\left(u_{n}\right), & x \in \mathbb{R}, t>-n, \\ u_{n}(x,-n)=\underline{u}_{1}(x,-n), & x \in \mathbb{R} .\end{cases}
$$

Then by Lemma 2.1 and the process of diagonalization, there exists a subsequence of $\left\{u_{n}\right\}$ converging in the space $C_{\text {loc }}^{2,1}(\mathbb{R} \times \mathbb{R})$. In fact, the limit of this subsequence is the entire solution as we desired. Obviously, $\underline{u}_{1}(x,-n)$ is the subsolution to 3.3 . Therefore,

$$
u_{n}(x,-n)=\underline{u}_{1}(x,-n) \leq u\left(x, t+n ; u_{n}(x,-n)\right), \quad t \geq-n .
$$

Specially, for any $\epsilon>0$, at $t=\epsilon-n$,

$$
u_{n}(x,-n) \leq u\left(x, \epsilon ; u_{n}(x,-n)\right) .
$$

Since $u\left(x, t+n+\epsilon ; u_{n}(x,-n)\right)$ satisfies

$$
\partial_{t} u_{n}=\partial_{x x} u_{n}+f\left(u_{n}\right) \quad \text { and } \quad u_{n}(x,-n+\epsilon)=u\left(x, \epsilon ; u_{n}(x,-n)\right) \text {, }
$$


then by the comparison theorem, $u_{n}(x, t) \leq u_{n}(x, t+\epsilon)$, for $t \geq-n$ and $x \in \mathbb{R}$. By the arbitrariness of $\epsilon$, one can conclude that for each $n \in \mathbb{N}, t \geq-n$ and $x \in \mathbb{R}, \partial_{t} u_{n} \geq 0$. By the convergence of $\left\{u_{n}\right\}$, then $\partial_{t} u>0$ or $\partial_{t} u \equiv 0$. Moreover, by the comparison theorem, for all $(x, t) \in \mathbb{R} \times \mathbb{R}$, we have

$$
\max \left\{\phi\left(x+c t+x_{6}\right), \phi\left(-x+c t+x_{6}\right)\right\}=\underline{u}_{1}(x, t) \leq u_{1}(x, t)<1 .
$$

Thus, on one hand, $\lim _{t \rightarrow+\infty} u_{1}(x, t)=1$ derived from (3.4). On the other hand, for any intervals referring to $x$ in $\mathbb{R}$, since $\lim _{t \rightarrow-\infty} \bar{u}_{1}(x, t)=0$ followed from $p_{1}(t) \rightarrow-\infty$ as $t \rightarrow-\infty$, then $\lim _{t \rightarrow-\infty} u_{1}(x, t)=0$. Therefore, by applying the strong maximum theorem, we arrive at $\partial_{t} u_{1}(x, t)>0$ for all $(x, t) \in \mathbb{R} \times \mathbb{R}$.

Now we deal with the case $(\mathrm{C} 2)$. According to $[13], u_{1}(x, t)$ is unique and satisfies (3.1), and the supersolution and subsolution for $t \leq 0$ are

$$
\bar{u}_{1}(x, t)=\phi\left(x+p_{2}(t)\right)+\phi\left(-x+p_{2}(t)\right), \quad \underline{u}_{1}(x, t)=\phi\left(x+p_{3}(t)\right)+\phi\left(-x+p_{3}(t)\right),
$$

where the functions $p_{2}(t)$ and $p_{3}(t)$ satisfy

$$
\left\{\begin{array}{l}
p_{2}^{\prime}(t)=c+M_{13} e^{\lambda_{1} p_{2}(t)}, \quad\left\{\begin{array}{l}
p_{3}^{\prime}(t)=c-M_{13} e^{\lambda_{1} p_{3}(t)}, \\
p_{2}(0) \leq 0,
\end{array} p_{3}(0) \leq \min \left\{0, \frac{1}{\lambda_{1}} \ln \left(\frac{c}{M_{13}}\right)\right\},\right.
\end{array}\right.
$$

and the expression of $M_{13}>0$ can be found in 13 .

It is easy to see that

$$
\liminf _{x \rightarrow \pm \infty} \underline{u}_{1}(x, 0)=\lim _{x \rightarrow \pm \infty}\left\{\phi\left(x+p_{3}(0)\right)+\phi\left(-x+p_{3}(0)\right)\right\}=1>\alpha
$$

which together with Lemma 2.3 implies that

$$
\lim _{t \rightarrow+\infty}\left\|u_{1}(\cdot, t)-1\right\|=0
$$

Eventually repeating the similar arguments as above, one can prove the monotonicity of $u_{1}(x, t)$ with respect to $t$.

Now we will consider the stability of the unique entire solution $u_{1}(x, t)$ obtained in Theorem 3.1 by using the method in [26], and obtain the following result.

Theorem 3.2. Suppose that $\int_{0}^{1} f(u) d u>0$. Then the unique entire solution $u_{1}(x, t)$ obtained in Theorem 3.1 is local exponential asymptotic stable.

Proof. To begin with, we introduce some notations. Due to $f \in C^{2}(\mathbb{R})$ and $f^{\prime}(0), f^{\prime}(1)<0$, there exists a $\theta>0$ such that $f^{\prime}(u)<0$ on $[-\theta, 2 \theta] \cup[1-2 \theta, 1+\theta]$. Therefore,

$$
v:=\max \left\{\max _{[-\theta, 2 \theta]} f^{\prime}(u), \max _{[1-2 \theta, 1+\theta]} f^{\prime}(u)\right\}<0 .
$$


Let $\phi(\xi)$ be the solution to 1.2 . When $\phi(x+c t)$ or $\phi(-x+c t) \in[\theta, 1-\theta]$, there is an $M_{14}>0$ such that

$$
\phi(x+c t)+\phi(-x+c t) \geq M_{14} .
$$

In the sequel of this paper, we still use these notations.

We first consider the case $(\mathrm{C} 1)$. For $t \geq 0$, we will prove that the following functions

$$
\begin{aligned}
& \bar{u}_{1}^{+}(x, t)=\min \left\{1, u_{1}\left(x, t+\gamma_{1}(t)\right)+q_{1}(t)\right\}, \\
& \underline{u}_{1}^{-}(x, t)=\max \left\{0, u_{1}\left(x, t-\gamma_{1}(t)\right)-q_{1}(t)\right\}
\end{aligned}
$$

are the supersolution and subsolution to (1.1) with the initial condition $u_{0}(x)=u_{1}(x, 0)$ respectively, where the function $q_{1}(t)$ is the solution to the following initial problem

$$
q_{1}^{\prime}(t)-v q_{1}(t)=0, \quad t>0, \quad q_{1}(0)=q_{10}
$$

$0 \leq q_{10} \leq \theta$ may be arbitrary, and the function $\gamma_{1}(t)$ is to be determined later.

Here, we mainly prove that the function $\bar{u}_{1}^{+}(x, t)$ is the supersolution, and the rest is similar. When $\bar{u}_{1}^{+}(x, t) \equiv 1$, the conclusion is obvious. Thus, we only consider $\bar{u}_{1}^{+}(x, t)=$ $u_{1}\left(x, t+\gamma_{1}(t)\right)+q_{1}(t)$. Firstly, when $u_{1}(x, t) \in[0, \theta]$ or $[1-\theta, 1]$, from (3.6), (3.7) and $\partial_{t} u_{1}(x, t)>0$, we can get

$$
\begin{aligned}
\partial_{t} \bar{u}_{1}^{+}-\partial_{x x} \bar{u}_{1}^{+}-f\left(\bar{u}_{1}^{+}\right) & =\gamma_{1}^{\prime}(t) \partial_{t} u_{1}+\partial_{t} u_{1}-\partial_{x x} u_{1}+q_{1}^{\prime}(t)-f\left(u_{1}+q_{1}(t)\right) \\
& \geq q_{1}^{\prime}(t)-v q_{1}(t)=0,
\end{aligned}
$$

where we need $\gamma_{1}^{\prime}(t)>0$.

On the other hand, when $u_{1}(x, t) \in[\theta, 1-\theta]$, due to $\partial_{t} u_{1}(x, t)>0$, there is a constant $M_{15}>0$ such that $\partial_{t} u_{1}(x, t) \geq M_{15}$. Thus it follows from $(2.4)$ and $(3.6)$ that

$$
\begin{aligned}
\partial_{t} \bar{u}_{1}^{+}-\partial_{x x} \bar{u}_{1}^{+}-f\left(\bar{u}_{1}^{+}\right) & =\gamma_{1}^{\prime}(t) \partial_{t} u_{1}+\partial_{t} u_{1}-\partial_{x x} u_{1}+q_{1}^{\prime}(t)-f\left(u_{1}+q_{1}(t)\right) \\
& \geq M_{15} \gamma_{1}^{\prime}(t)+v q_{1}(t)-w q_{1}(t)=0,
\end{aligned}
$$

where we need the function $\gamma_{1}(t)$ satisfying

$$
\gamma_{1}^{\prime}(t)=\frac{w-v}{M_{15}} q_{10} e^{v t}, \quad t>0, \quad \gamma_{1}(0)=q_{10} .
$$

Solving the equations (3.7) and (3.8) yields that $q_{1}(t)=q_{10} e^{v t}$ and $\gamma_{1}(t)=q_{10}\left(1+\gamma_{0}-\right.$ $\left.\gamma_{0} e^{v t}\right)$, where $\gamma_{0}=\frac{v-w}{M_{15} v}>0$, since $v<0$ and $w>0$. Also since $\partial_{t} u_{1}(x, t)>0$, then

$$
u_{1}(x, 0) \leq u_{1}\left(x, q_{10}\right)+q_{10}=\bar{u}_{1}^{+}(x, 0) .
$$

Hence, $\bar{u}_{1}^{+}(x, t)$ is the supersolution of (1.1) with the initial condition $u_{0}(x)=u_{1}(x, 0)$. Similarly, one can prove that $\underline{u}_{1}^{-}(x, t)$ is the subsolution of 1.1 with the initial condition $u_{0}(x)=u_{1}(x, 0)$. 
Now we prove the local stability of the entire solution $u_{1}(x, t)$. For any given $\epsilon>0$, from Lemma 2.1, there exists a positive constant $\delta_{1} \leq \frac{\epsilon}{2 M_{2}}$, such that for any $\left|t_{2}\right| \leq \delta_{1}$ and $t \in\left[1+t_{2},+\infty\right)$,

$$
\left\|u_{1}\left(\cdot, t+t_{2}\right)-u_{1}(\cdot, t)\right\|=\left\|\partial_{t} u_{1}\left(\cdot, t+t^{*}\right)\right\|\left|t_{2}\right| \leq \frac{\epsilon}{2}
$$

where $t^{*} \in\left(-t_{2}, t_{2}\right)$. Choose $q_{10}=\delta \leq \min \left\{\frac{\delta_{1}}{1+\gamma_{0}}, \frac{\epsilon}{2}\right\}$, and the initial function $u_{0}(x)$ satisfies $\left\|u_{0}(\cdot)-u_{1}(\cdot, 0)\right\|<\delta$. Since $\partial_{t} u_{1}(x, t)>0$, then

$$
u_{1}(x, 0)+\delta \leq u_{1}(x, \delta)+\delta, \quad u_{1}(x,-\delta)-\delta \leq u_{1}(x, 0)-\delta
$$

Therefore, for all $x \in \mathbb{R}$, we have

$$
u_{1}(x,-\delta)-\delta \leq u_{1}(x, 0)-\delta \leq u_{0}(x) \leq u_{1}(x, 0)+\delta \leq u_{1}(x, \delta)+\delta .
$$

Thus $\bar{u}_{1}^{+}(x, t)$ and $\underline{u}_{1}^{-}(x, t)$ are also the supersolution and subsolution of $(1.1)$ with the initial condition $u_{0}(x)$. Consequently,

$$
\begin{aligned}
& \max \left\{0, u_{1}\left(x, t-\delta\left(1+\gamma_{0}-\gamma_{0} e^{v t}\right)\right)-\delta e^{v t}\right\} \\
\leq & u\left(x, t ; u_{0}\right) \leq \min \left\{1, u_{1}\left(x, t+\delta\left(1+\gamma_{0}-\gamma_{0} e^{v t}\right)\right)+\delta e^{v t}\right\} .
\end{aligned}
$$

By noting that $\delta\left(1+\gamma_{0}-\gamma_{0} e^{v t}\right) \leq \delta_{1}$, it follows from $(3.9)$ that

$$
u_{1}(x, t)-\epsilon \leq u\left(x, t ; u_{0}\right) \leq u_{1}(x, t)+\epsilon \text {. }
$$

In a word, for any $\epsilon>0$, there exists a $\delta>0$, when $\left\|u_{0}(\cdot)-u_{1}(\cdot, 0)\right\|<\delta$, then

$$
\left\|u\left(\cdot, t ; u_{0}\right)-u_{1}(\cdot, t)\right\| \leq \epsilon, \quad t \geq 0
$$

which means that the entire solution $u_{1}(x, t)$ is local stable.

In the end, we will show that the entire solution $u_{1}(x, t)$ is local exponential asymptotic stable. First of all, from (2.3) and (3.4), there is a $T_{3}>0$ with $c T_{3}+x_{6}>0$ such that for any $t \geq T_{3}$, we have

$$
0 \leq 1-u_{1}(x, t) \leq 1-\underline{u}_{1}(x, t) \leq M_{6} e^{\mu_{2}\left(|x|+c t+x_{6}\right)} \leq M_{6} e^{\mu_{2} c t} .
$$

The next step is to prove that

$$
u_{1}\left(x, t-\delta\left(1+\gamma_{0}-\gamma_{0} e^{v t}\right)\right)-\delta e^{v t}>0
$$

holds for large $t$, which implies $\underline{u}_{1}^{-}(x, t)=u_{1}\left(x, t-\delta\left(1+\gamma_{0}-\gamma_{0} e^{v t}\right)\right)$. Indeed, we remark that

$$
\partial_{t}\left(u_{1}\left(x, t-q_{10}\left(1+\gamma_{0}-\gamma_{0} e^{v t}\right)\right)-q_{10} e^{v t}\right)=\left(1+q_{10} \gamma_{0} v e^{v t}\right) \partial_{t} u_{1}-q_{10} v e^{v t}
$$


Since $\gamma_{0}=\frac{v-w}{M_{15 v}}$, then

$$
1+q_{10} \gamma_{0} v e^{v t}=1-q_{10} \frac{w-v}{M_{15}} e^{v t} .
$$

Hence we can choose a constant $T_{4} \geq \max \left\{0, \frac{1}{v} \ln \frac{M_{15}}{(w-v) \theta}\right\}$ such that when $t \geq T_{4}$, by noting that $w>0, v<0, M_{15}>0$ as well as $0 \leq q_{10} \leq \theta$, we have

$$
q_{10} \frac{w-v}{M_{15}} e^{v t} \leq \theta \frac{w-v}{M_{15}} e^{v t} \leq \theta \frac{w-v}{M_{15}} e^{v T_{4}} \leq 1 .
$$

Therefore, when $t \geq T_{4}$, from $v<0$ and $\partial_{t} u_{1}(x, t)>0$ as well as (3.13), we can obtain

$$
\partial_{t}\left(u_{1}\left(x, t-q_{10}\left(1+\gamma_{0}-\gamma_{0} e^{v t}\right)\right)-q_{10} e^{v t}\right)>0 .
$$

Moreover, by noting $\lim _{t \rightarrow+\infty} \underline{u}_{1}\left(x, t-q_{10}\left(1+\gamma_{0}-\gamma_{0} e^{v t}\right)\right)=1$, thus, there is a constant $T_{5} \geq \max \left\{T_{3}, T_{4}, \delta\left(1+\gamma_{0}\right), \delta\left(1+\gamma_{0}\right)-\frac{x_{6}}{c}\right\}$ such that for $t \geq T_{5}, 3.12$ holds.

Therefore, for $t \geq T_{5} \geq \delta\left(1+\gamma_{0}\right)$, it follows from (3.10) and (3.12) that

$$
\begin{aligned}
\left|1-u\left(x, t ; u_{0}\right)\right| & \leq\left|1-u_{1}\left(x, t-\delta\left(1+\gamma_{0}-\gamma_{0} e^{v t}\right)\right)+\delta e^{v t}\right| \\
& \leq M_{6} e^{\mu_{2}\left(|x|+c t-c \delta\left(1+\gamma_{0}-\gamma_{0} e^{v t}\right)+x_{6}\right)}+\delta e^{v t} \\
& \leq M_{6} e^{\mu_{2} c t}+\delta e^{v t} .
\end{aligned}
$$

Thus, when $\left\|u_{0}(\cdot)-u_{1}(\cdot, 0)\right\|<\delta$, for $t \geq T_{5}$, from (3.11) and (3.14), we have

$$
\left\|u\left(\cdot, t ; u_{0}\right)-u_{1}(\cdot, t)\right\| \leq\left\|1-u_{1}(\cdot, t)\right\|+\left\|1-u\left(\cdot, t ; u_{0}\right)\right\| \leq 2 M_{6} e^{\mu_{2} c t}+\delta e^{v t} .
$$

Hence $u_{1}(x, t)$ is local exponential asymptotic stable.

Next we discuss the stability of $u_{1}(x, t)$ under the case $(\mathrm{C} 2)$. The proof of the local stability is similar, we only need to consider the local exponential stability. By directly calculating (3.5), we know that

$$
p_{3}(t)=p_{3}(0)+c t-\frac{1}{\lambda_{1}} \ln \left\{1-\frac{M_{13}}{c} e^{\lambda_{1} p_{3}(0)}\left(1-e^{c \lambda_{1} t}\right)\right\} .
$$

Set

$$
g(t)=\frac{1}{\lambda_{1}} \ln \left\{1-\frac{M_{13}}{c} e^{\lambda_{1} p_{3}(0)}\left(1-e^{c \lambda_{1} t}\right)\right\} .
$$

Then

$$
g^{\prime}(t)=\frac{c M_{13} e^{\lambda_{1} p_{3}(0)} e^{\lambda_{1} t}}{c-M_{13} e^{\lambda_{1} p_{3}(0)}+M_{13} e^{\lambda_{1} p_{3}(0)} e^{c \lambda_{1} t}}>0,
$$

since $p_{3}(0)<\frac{1}{\lambda_{1}} \ln \frac{c}{M_{13}}$. Thus, $g(t)<g(\infty)=\frac{1}{\lambda_{1}} \ln \left\{1-\frac{M_{13}}{c} e^{\lambda_{1} p_{3}(0)}\right\}:=x_{7}$. Consequently, for $t \leq 0, p_{3}(t)>c t+x_{8}$, where $x_{8}:=p_{3}(0)-x_{7}$. As a result, for $t \leq 0$,

$$
\underline{u}_{1}(x, t)>\max \left\{\phi\left(x+c t+x_{8}\right), \phi\left(-x+c t+x_{8}\right)\right\} .
$$


Then by the comparison theorem, for $(x, t) \in \mathbb{R} \times \mathbb{R}$, we have

$$
\underline{u}(x, t) \geq \max \left\{\phi\left(x+c t+x_{8}\right), \phi\left(-x+c t+x_{8}\right)\right\} .
$$

Thus in the case $(\mathrm{C} 2)$, we also get an inequality similar to 3.4 . The rest of the proof is similar.

Remark 3.3. Under the cases $(\mathrm{C} 1)$ and $(\mathrm{C} 2)$, since $\int_{0}^{1} f(u) d u>0$, the entire solution $u_{1}(x, t)$ found in Theorem 3.1 satisfies

$$
\lim _{t \rightarrow+\infty}\left\|u_{1}(\cdot, t)-1\right\|=0
$$

In fact, this conclusion coincides with that in [28]. Thus the super-sub solution method is a valid way to simplify the proof.

Remark 3.4. Under the case (C1), since the subsolution is

$$
\underline{u}_{1}(x, t)=\max \left\{\phi\left(x+c t+x_{6}\right), \phi\left(-x+c t+x_{6}\right)\right\},
$$

the existence, uniqueness and stability of entire solutions to (1.1) can be found in [26] as well. Here in order to prove the local asymptotic stability of entire solutions, the different supersolution and subsolution are constructed compared with [26], and the proof is also more simple.

Remark 3.5. For 1.3$)$, that is, $f(u)=u(1-u)(u-\alpha), \alpha \in(0,1)$, it is easy to see that $\int_{0}^{1} f(s) d s=(1-2 \alpha) / 12$ and $f^{\prime}(0)=-\alpha, f^{\prime}(1)=-1+\alpha$. Obviously,

$$
f^{\prime}(0) \lesseqgtr f^{\prime}(1) \quad \text { if and only if } \quad \int_{0}^{1} f(u) d u \lesseqgtr 0 \quad \text { if and only if } \quad \alpha \gtreqless \frac{1}{2} \text {. }
$$

Therefore if $\int_{0}^{1} f(u) d u>0$, then $f^{\prime}(0)>f^{\prime}(1)$, which implies that only the case $(\mathrm{C} 1)$ will occur.

For the case $\int_{0}^{1} f(u) d u<0$, the authors in 3 had found the entire solution $u_{2}(x, t)$ to (1.1). Now we will discuss the long time behavior and the local exponential asymptotic stability of the entire solution in the following theorem.

Theorem 3.6. Assume that $\int_{0}^{1} f(u) d u<0$ and $\phi$ is the solution to (1.2), then (1.1) admits a unique entire solution $u_{2}(x, t)$ satisfying $\partial_{t} u_{2}(x, t)<0, u_{2}(x, t)=u_{2}(-x, t)$, $0<u_{2}(x, t)<1$, and for $(x, t) \in \mathbb{R} \times(-\infty,-4 M \phi(0)]$,

$$
u_{2}\left(x, t+h_{1}(t)\right)<\phi(-x+c t) \phi(x+c t)<u_{2}\left(x, t-h_{1}(t)\right)
$$

where $h_{1}(t)=4 M \phi(c t), \lim _{t \rightarrow+\infty}\left\|u_{2}(\cdot, t)\right\|=0$,

$$
\lim _{t \rightarrow-\infty}\left\{\sup _{x \geq 0}\left|u_{2}(x, t)-\phi(-x+c t)\right|+\sup _{x \leq 0}\left|u_{2}(x, t)-\phi(x+c t)\right|\right\}=0 .
$$

Moreover, the unique entire solution $u_{2}(x, t)$ is local exponential asymptotic stable. 
Proof. Firstly, it follows from [3] that

$$
\lim _{t \rightarrow-\infty} \sup _{x \in \mathbb{R}}\left|u_{2}(x, t)-\phi(x+c t) \phi(-x+c t)\right|=0,
$$

which implies that

$$
\begin{aligned}
& \lim _{t \rightarrow-\infty} \sup _{x \geq 0}\left|u_{2}(x, t)-\phi(-x+c t)\right| \\
\leq & \lim _{t \rightarrow-\infty} \sup _{x \geq 0}\left|u_{2}(x, t)-\phi(-x+c t) \phi(x+c t)\right|+\lim _{t \rightarrow-\infty} \sup _{x \geq 0}|\phi(-x+c t)||1-\phi(x+c t)|=0 .
\end{aligned}
$$

Similarly, $\lim _{t \rightarrow-\infty} \sup _{x \leq 0}\left|u_{2}(x, t)-\phi(x+c t)\right|=0$. Thus, the entire solution $u_{2}(x, t)$ satisfies 3.15 .

Since $\int_{0}^{1} f(u) d u<0$, then the wave speed $c<0$, and at the same time, according to [3], the entire solution $u_{2}(x, t)$ satisfies for $t \leq-4 M \phi(0)<0$,

$$
u_{2}\left(x, t+h_{1}(t)\right)<\phi(-x+c t) \phi(x+c t)<u\left(x, t-h_{1}(t)\right) .
$$

Hence, for any $\tau \leq-4 M \phi(0)<0$, we have

$$
u_{2}\left(x, \tau+h_{1}(\tau)\right)<\phi(x+c \tau),
$$

which together with the comparison theorem yields that for all $t>\tau, u_{2}\left(x, t+h_{1}(\tau)\right)<$ $\phi(x+c t)$. Since $h_{1}(t)=4 M \phi(c t)$, setting $\tau \rightarrow-\infty$ leads to $u_{2}(x, t) \leq \phi(x+c t)$. Similarly, $u_{2}(x, t) \leq \phi(-x+c t)$. Thus, for all $(x, t) \in \mathbb{R} \times \mathbb{R}$,

$$
u_{2}(x, t) \leq \min \{\phi(x+c t), \phi(-x+c t)\} .
$$

Specially, $u_{2}(x, 0) \leq \min \{\phi(x), \phi(-x)\}$, which implies that $\lim _{x \rightarrow \pm \infty} u_{2}(x, 0)=0<\alpha$. Hence, due to Lemma 2.3. $\lim _{t \rightarrow+\infty}\left\|u_{2}(\cdot, t)\right\|=0$.

Now, repeating the processes of the proof in Theorem 3.1, there are some constants $\alpha_{1}<\alpha<\alpha_{2}$ such that $f \neq 0$ in $\left(0, \alpha_{1}\right] \cup\left[\alpha_{2}, 1\right)$. Then from the monotonicity of $\phi$, there exists a positive constant $\widehat{x}$ such that $\phi(-\widehat{x}) \leq \alpha_{1}$. Set

$$
l_{2}(t)=-c t-\widehat{x}, \quad m_{2}(t)=c t+\widehat{x},
$$

then for any $t \leq 0$, obviously $m_{2}(t) \geq 0 \geq l_{2}(t)$, and according to $(3.16), u_{2}(x, t) \leq \alpha_{1}$ for any $x \in\left(-\infty, l_{2}(t)\right] \cup\left[m_{2}(t),+\infty\right)$. On the other hand, according to 3.15 , for any $\varepsilon>0$, there exists a $T_{13}<0$ such that for any $t \leq T_{13}$,

$$
\sup _{x \geq 0}\left|u_{2}(x, t)-\phi(-x+c t)\right| \leq \varepsilon, \quad \sup _{x \leq 0}\left|u_{2}(x, t)-\phi(x+c t)\right| \leq \varepsilon,
$$

which together with the monotonicity of $\phi$ implies that

$$
u_{2}(x, t) \geq \phi\left(-m_{2}(t)+d_{2}+c t\right)-\varepsilon \geq \alpha_{2}
$$


holds for $x \leq-m_{2}(t)+d_{2}=-c t+\widehat{x}+d_{2}$ with some sufficiently large positive constant $d_{2}$. Similarly, $u_{2}(x, t) \geq \alpha_{2}$ for $x \geq l_{2}(t)+d_{2}$. Since there is a $T_{14}<0$ satisfying $l_{2}(t)+d_{2} \leq 0 \leq m_{2}(t)-d_{2}$ for any $t \leq T_{14}$, if we choose $T_{2} \leq \min \left\{T_{13}, T_{14}\right\}$, then $u_{2}(x, t) \geq \alpha_{2}$ for any $t \leq T_{2}$ and $x \in\left[l_{2}(t)+d_{2}, m_{2}(t)-d_{2}\right]$. Therefore, the entire solution $u_{2}(x, t)$ satisfies the condition $\mathbb{M}^{-}$, and from the proof in the papers 3,26 , it is unique up to a space-time translation.

Finally, we are going to prove the local asymptotic exponential stability of $u_{2}(x, t)$. First of all, for all $(x, t) \in \mathbb{R} \times \mathbb{R}$, from Theorem 1 in $[3], \partial_{t} u_{2}(x, t)<0$. Thus there exists a negative constant $M_{16}$ such that when $u_{2}(x, t) \in[\theta, 1-\theta], \partial_{t} u_{2}(x, t) \leq M_{16}<0$. Similarly, it is not hard to prove that the following two functions

$$
\begin{aligned}
& \bar{u}_{2}^{+}(x, t)=\min \left\{1, u_{2}\left(x, t-\gamma_{2}(t)\right)+q_{1}(t)\right\}, \\
& \underline{u}_{2}^{-}(x, t)=\max \left\{0, u_{2}\left(x, t+\gamma_{2}(t)\right)-q_{1}(t)\right\}
\end{aligned}
$$

are the supersolution and subsolution of $(1.1)$ with the initial condition $u_{0}(x)=u_{2}(x, 0)$, respectively. At this time, the function $q_{1}(t)$ also satisfies (3.7), while the function $\gamma_{2}(t)$ satisfies

$$
\gamma_{2}^{\prime}(t)=\frac{w-v}{-M_{16}} q_{10} e^{v t}, \quad t>0, \quad \gamma_{2}(0)=q_{10},
$$

where the parameters $q_{10}, \theta$ are defined in the above, as well as $w$ and $v$ are defined in (2.4) and (3.6) separately. Then from the proof in Theorem 3.2 or [26], we know that $u_{2}(x, t)$ is local Lyapunov stable. Now we prove the local exponential asymptotic stability of $u_{2}(x, t)$. With the similar proof, it is not hard to see that there is a constant $T_{6}$ such that for $t \geq T_{6}, \bar{u}_{2}^{+}(x, t)=u_{2}\left(x, t-\gamma_{2}(t)\right)+q_{1}(t)$. By choosing $T_{7}=\max \left\{T_{6}, \delta\left(1+\widetilde{\gamma}_{0}\right)\right\}$, where $\widetilde{\gamma}_{0}=\frac{w-v}{M_{16} v}>0$, and noting $c<0$, then when $t \geq T_{7}$, with the help of 2.3 and (3.16), we finally arrive at

$$
\begin{aligned}
\left\|u\left(x, t ; u_{0}\right)-u_{2}(x, t)\right\| & \leq\left\|u_{2}(x, t)\right\|+\left\|u\left(x, t ; u_{0}\right)\right\| \\
& \leq u_{2}(x, t)+u_{2}\left(x, t-\delta\left(1+\widetilde{\gamma}_{0}-\widetilde{\gamma}_{0} e^{v t}\right)\right)+\delta e^{v t} \\
& \leq 2 M_{8} e^{\lambda_{1} c t}+\delta e^{v t}
\end{aligned}
$$

which implies the local exponential asymptotic stability of $u_{2}(x, t)$. Thus we have finished the proof.

4. The continuous dependence of the entire solution of Allen-Cahn equation

In this section we will prove the continuity of the entire solution to $\sqrt{1.3}$ in $\alpha$. Let $\phi_{\alpha}$ be the traveling front solution to $(1.3)$, which in fact satisfies

$$
\begin{gathered}
\phi_{\alpha}^{\prime \prime}(\xi)-c \phi_{\alpha}^{\prime}(\xi)+\phi_{\alpha}(\xi)\left(1-\phi_{\alpha}(\xi)\right)\left(\phi_{\alpha}(\xi)-\alpha\right)=0, \\
\lim _{\xi \rightarrow-\infty} \phi_{\alpha}(\xi)=0, \quad \lim _{\xi \rightarrow+\infty} \phi_{\alpha}(\xi)=1, \quad \phi_{\alpha}^{\prime}(\xi)>0 .
\end{gathered}
$$


In the rest of this section, we only consider $\alpha \in(0,1 / 2)$. For (4.1), the corresponding $\lambda_{1}=\sqrt{2} / 2$ and

$$
\max _{u \in[0,1]}(u(1-u)(u-\alpha))^{\prime}=\frac{1}{3}\left(1-\alpha+\alpha^{2}\right), \quad \max _{u \in[0,1]}(u(1-u)(u-\alpha))^{\prime \prime}=2(1+\alpha) .
$$

Let $u_{k}(x, t):=u_{\alpha_{k}}(x, t)$ be the entire solution to 1.3$)$ with the parameter $\alpha_{k}$, and the sequence $\left\{\alpha_{k}\right\}$ converges to $\alpha \in(0,1 / 2)$, as $k$ converges to $+\infty$. For any $\alpha \in(0,1 / 2)$, choose $M_{0}=1$ and $M_{1}=3$ in Lemma 2.1, there is a subsequence of $\left\{u_{k}(x, t)\right\}$ which converges to $\widetilde{u}_{\alpha}(x, t)$ uniformly in any compact sets of $\mathbb{R} \times \mathbb{R}$, which is also an entire solution to 1.3$)$. Moreover, $\widetilde{u}_{\alpha}(x, t)$ satisfies

$$
\max \left\{\phi_{\alpha}\left(x+c t+x_{6}\right), \phi_{\alpha}\left(-x+c t+x_{6}\right)\right\} \leq \widetilde{u}_{\alpha}(x, t) \leq \min \{\overline{\widetilde{u}}(x, t), 1\}
$$

where $\overline{\widetilde{u}}(x, t)=\phi\left(x+p_{1}(t)\right)+\phi\left(-x+p_{1}(t)\right), x_{6}$ and $p_{1}(t)$ are defined in 3.2 in Theorem 3.1 . For convenience, choose $p_{1}(0)=0$, then

$$
x_{6}=-\frac{1}{\lambda_{1}} \ln \left(1+\frac{M_{12}}{c}\right), \quad p_{1}(t)=c t-\frac{1}{\lambda_{1}} \ln \left\{1+\frac{M_{12}}{c}\left(1-e^{\lambda_{1} c t}\right)\right\} .
$$

Furthermore, from [13], we know that for some positive constant $M_{17}$,

$$
\left|p_{1}(t)-c t-x_{6}\right| \leq M_{17} e^{\lambda_{1} c t}, \quad t<0 \text {. }
$$

In addition, if the reaction item $f(u)$ in 1.1 is $u(1-u)(u-\alpha)$, from 3,13 , the entire solution $u_{1}(x, t)$ of 1.1 is the limit of $u_{n}(x, t)$, which actually satisfies

$$
\begin{aligned}
\partial_{t} u_{n} & =\partial_{x x} u_{n}+u_{n}\left(1-u_{n}\right)\left(u_{n}-\alpha\right), & & x \in \mathbb{R}, t>-n, \\
u_{n}(x,-n) & =\max \left\{\phi_{\alpha}\left(x-c n+x_{6}\right), \phi_{\alpha}\left(-x-c n+x_{6}\right)\right\}, & & x \in \mathbb{R} .
\end{aligned}
$$

Thus,

$$
\left|\widetilde{u}_{\alpha}(x,-n)-u_{n}(x,-n)\right| \leq \begin{cases}\overline{\widetilde{u}}(x,-n)-\phi_{\alpha}\left(x-c n+x_{6}\right) & \text { if } x \geq 0 \\ \overline{\widetilde{u}}(x,-n)-\phi_{\alpha}\left(-x-c n+x_{6}\right) & \text { if } x<0 .\end{cases}
$$

In what follows, we will estimate $\widetilde{u}_{\alpha}(x, t)$ and $u_{1}(x, t)$ at any $(x, t) \in \mathbb{R} \times \mathbb{R}$. To finish it, by noting the convergence of $\left\{u_{n}\right\}$, we need to estimate $\widetilde{u}_{\alpha}(x, t)$ and $u_{n}(x, t)$. Let $v_{n}(x, t)=\widetilde{u}_{\alpha}(x, t)-u_{n}(x, t)$, then it solves

$$
\begin{aligned}
& \partial_{t} v_{n}=\partial_{x x} v_{n}+\widetilde{u}_{\alpha}\left(1-\widetilde{u}_{\alpha}\right)\left(\widetilde{u}_{\alpha}-\alpha\right)-u_{n}\left(1-u_{n}\right)\left(u_{n}-\alpha\right), \\
& v(x,-n) \leq \begin{cases}\overline{\widetilde{u}}(x,-n)-\phi_{\alpha}\left(x-c n+x_{6}\right) & \text { if } x \geq 0, \\
\overline{\widetilde{u}}(x,-n)-\phi_{\alpha}\left(-x-c n+x_{6}\right) & \text { if } x<0 .\end{cases}
\end{aligned}
$$


Since $\widetilde{u}_{\alpha}\left(1-\widetilde{u}_{\alpha}\right)\left(\widetilde{u}_{\alpha}-\alpha\right)-u_{n}\left(1-u_{n}\right)\left(u_{n}-\alpha\right) \leq \frac{1}{3}\left(1-\alpha+\alpha^{2}\right) v_{n}$, then, estimating $v_{n}(x, t)$ is changed into estimating the solution $w_{n}(x, t)$ of the following linear parabolic equation

$$
\begin{gathered}
\partial_{t} w_{n}=\partial_{x x} w_{n}+\frac{1}{3}\left(1-\alpha+\alpha^{2}\right) w_{n}, \\
w_{n}(x,-n)= \begin{cases}\overline{\widetilde{u}}(x,-n)-\phi_{\alpha}\left(x-c n+x_{6}\right) & \text { if } x \geq 0, \\
\overline{\widetilde{u}}(x,-n)-\phi_{\alpha}\left(-x-c n+x_{6}\right) & \text { if } x<0 .\end{cases}
\end{gathered}
$$

The solution $w_{n}(x, t)$ can be presented as

$$
\begin{aligned}
w_{n}(x, t)= & \frac{1}{\sqrt{4 \pi(t+n)}} e^{\frac{1}{3}\left(1-\alpha+\alpha^{2}\right)(t+n)} \int_{\mathbb{R}} w_{n}(y,-n) e^{-\frac{(x-y)^{2}}{4(t+n)}} d y \\
= & \frac{1}{\sqrt{4 \pi(t+n)}} e^{\frac{1}{3}\left(1-\alpha+\alpha^{2}\right)(t+n)} \int_{0}^{+\infty}\left(\overline{\widetilde{u}}(y,-n)-\phi_{\alpha}\left(y-c n+x_{6}\right)\right) e^{-\frac{(x-y)^{2}}{4(t+n)}} d y \\
& +\frac{1}{\sqrt{4 \pi(t+n)}} e^{\frac{1}{3}\left(1-\alpha+\alpha^{2}\right)(t+n)} \int_{-\infty}^{0}\left(\overline{\widetilde{u}}(y,-n)-\phi_{\alpha}\left(-y-c n+x_{6}\right)\right) e^{-\frac{(x-y)^{2}}{4(t+n)}} d y \\
:= & I_{1}(x, t)+I_{2}(x, t) .
\end{aligned}
$$

We firstly consider the integral $I_{1}(x, t)$. Let $z=y-c n+x_{6}$, then

$$
\begin{array}{r}
I_{1}(x, t)=\frac{1}{\sqrt{4 \pi(t+n)}} e^{\frac{1}{3}\left(1-\alpha+\alpha^{2}\right)(t+n)} \int_{-c n+x_{6}}^{+\infty}\left[\phi_{\alpha}\left(-z-c n-x_{6}+p_{1}(-n)\right)\right. \\
\left.\quad+\phi_{\alpha}\left(z-c n+x_{6}+p_{1}(-n)\right)-\phi_{\alpha}(z)\right] e^{-\frac{\left(x-z-c n+x_{6}\right)^{2}}{4(t+n)}} d z .
\end{array}
$$

In order to further estimate $I_{1}$, since $(1.3)$ is the special case of (1.1), then $\phi_{\alpha}$ satisfies (2.3). Combining 2.3 and 4.2 gives

$$
\begin{aligned}
I_{1}(x, t) \leq & \frac{1}{\sqrt{4 \pi(t+n)}} e^{\frac{1}{3}\left(1-\alpha+\alpha^{2}\right)(t+n)} \\
& \times \int_{-c n+x_{6}}^{+\infty}\left(M_{8} e^{\lambda_{1}\left(-z-c n+x_{6}+p_{1}(-n)\right)}+M_{17} \sup _{\mathbb{R}} \phi^{\prime} e^{-c \lambda_{1} n}\right) e^{-\frac{\left(x-z-c n+x_{6}\right)^{2}}{4(t+n)}} d z \\
\leq & \frac{1}{\sqrt{4 \pi(t+n)}} e^{\frac{1}{3}\left(1-\alpha+\alpha^{2}\right) t} e^{\left[\frac{1}{3}\left(1-\alpha+\alpha^{2}\right)-c \lambda_{1}\right] n} \\
& \times \int_{-c n+x_{6}}^{+\infty}\left(M_{8} e^{\lambda_{1}\left(-z+x_{6}+p_{1}(-n)\right)}+M_{17} \sup _{\mathbb{R}} \phi^{\prime}\right) e^{-\frac{\left(x-z-c n+x_{6}\right)^{2}}{4(t+n)}} d z \\
\leq & M_{18} e^{\frac{1}{3}\left(1-\alpha+\alpha^{2}\right) t} e^{\left[\frac{1}{3}\left(1-\alpha+\alpha^{2}\right)-c \lambda_{1}\right] n},
\end{aligned}
$$

where $M_{18} \geq M_{17} \sup _{\mathbb{R}} \phi^{\prime}+M_{8} e^{\lambda_{1}\left(x_{6}-\frac{1}{\lambda_{1}} \ln \left\{1+\frac{M_{12}}{c}\left(1-e^{-\lambda_{1} c n}\right)\right\}\right)}$.

Moreover, by noting $c=\sqrt{2}(1 / 2-\alpha)$ and $\lambda_{1}=\sqrt{2} / 2$, for $\alpha \in(0, \sqrt{6} / 2-1)$,

$$
\frac{1}{3}\left(1-\alpha+\alpha^{2}\right)-c \lambda_{1}=\frac{1}{6}\left(2 \alpha^{2}+4 \alpha-1\right)<0 .
$$


Therefore, for any fixed $(x, t) \in \mathbb{R} \times \mathbb{R}$ and $\alpha \in(0, \sqrt{6} / 2-1), \lim _{n \rightarrow+\infty} I_{1}(x, t)=0$. Similarly, $\lim _{n \rightarrow+\infty} I_{2}(x, t)=0$. In a word, as $n$ converges to $+\infty$, for $\alpha \in(0, \sqrt{6} / 2-1)$, $w_{n}$ converges to 0 , thus $\lim _{n \rightarrow+\infty} v_{n}=0$. Hence $\widetilde{u}_{\alpha}(x, t)=u_{1}(x, t)$. Therefore,

Theorem 4.1. The entire solution $u_{1}(x, t)$ of $(1.3)$ is continuous in $\alpha \in(0, \sqrt{6} / 2-1)$.

\section{References}

[1] D. G. Aronson and H. F. Weinberger, Nonlinear diffusion in population genetics, combustion, and nerve pulse propagation, Partial Differential Equations and Related Topics (Program, Tulane Univ., New Orleans, La., 1974), 5-49, Lecture Notes in Mathematics 446, Springer, Berlin, 1975.

[2] _ Multidimensional nonlinear diffusion arising in population genetics, Adv. in Math. 30 (1978), no. 1, 33-76.

[3] X. Chen and J.-S. Guo, Existence and uniqueness of entire solutions for a reactiondiffusion equation, J. Differential Equations 212 (2005), no. 1, 62-84.

[4] Y.-Y. Chen, J.-S. Guo, H. Ninomiya and C.-H. Yao, Entire solutions originating from monotone fronts to the Allen-Cahn equation, Phys. D 378/379 (2018), 1-19.

[5] H. Cohen, Nonlinear diffusion problems, Studies in Applied Mathematics, 27-64, MAA Studies in Mathematics 7, Math. Assoc. of Amer. (distributed by PrenticeHall, Englewood Cliffs, N.J.), 1971.

[6] P. C. Fife, Mathematical Aspects of Reacting and Diffusing Systems, Lecture Notes in Biomathematics 28, Springer-Verlag, Berlin, 1979.

[7] _ Long time behavior of solutions of bistable nonlinear diffusion equations, Arch. Rational Mech. Anal. 70 (1979), no. 1, 31-46.

[8] P. C. Fife and J. B. McLeod, The approach of solutions of nonlinear diffusion equations to travelling front solutions, Arch. Rational Mech. Anal. 65 (1977), no. 4, 335361.

[9] _ A phase plane discussion of convergence to travelling fronts for nonlinear diffusion, Arch. Rational Mech. Anal. 75 (1981), no. 4, 281-314.

[10] R. A. Fisher, The wave of advance of advantageous genes, Ann. Eugenics 7 (1937), no. $4,355-369$. 
[11] Y. Fukao, Y. Morita and H. Ninomiya, Some entire solutions of the Allen-Cahn equation, Proceedings of Third East Asia Partial Differential Equation Conference, Taiwanese J. Math. 8 (2004), no. 1, 15-32.

[12] T. Gallay and E. Risler, A variational proof of global stability for bistable travelling waves, Differential Integral Equations 20 (2007), no. 8, 901-926.

[13] J.-S. Guo and Y. Morita, Entire solutions of reaction-diffusion equations and an application to discrete diffusive equations, Discrete Contin. Dyn. Syst. 12 (2005), no. 2, 193-212.

[14] F. Hamel and N. Nadirashvili, Entire solutions of the KPP equation, Comm. Pure Appl. Math. 52 (1999), no. 10, 1255-1276.

[15] _ Travelling fronts and entire solutions of the Fisher-KPP equation in $R^{N}$, Arch. Ration. Mech. Anal. 157 (2001), no. 2, 91-163.

[16] Y. Kan-on, Existence of standing waves for competition-diffusion equations, Japan J. Indust. Appl. Math. 13 (1996), no. 1, 117-133.

[17] A. Kolmogoroff, I. Petrovsky and N. Piscounoff, Étude de Íequation de la diffusion avec croissance de la quantité de matière et son application à unprobleme biologique, Bull. Univ. Moskou, Ser. Internat., Sec. A 1 (1937), no. 6, 1-25.

[18] C. D. Levermore and J. X. Xin, Multidimensional stability of traveling waves in a bistable reaction-diffusion equation II, Comm. Partial Differential Equations 17 (1992), no. 11-12, 1901-1924.

[19] H. Matano and P. Poláčik, An entire solution of a bistable parabolic equation on $\mathbb{R}$ with two colliding pulses, J. Funct. Anal. 272 (2017), no. 5, 1956-1979.

[20] Y. Morita and H. Ninomiya, Entire solutions with merging fronts to reaction-diffusion equations, J. Dynam. Differential Equations 18 (2006), no. 4, 841-861.

[21] J. Nagumo, S. Yoshizawa and S. Arimoto, Bistable transmission lines, IEEE Trans. Circuit Theory 12 (1965), no. 3, 400-412.

[22] T. Ogiwara and H. Matano, Monotonicity and convergence results in order-preserving systems in the presence of symmetry, Discrete Contin. Dynam. Systems 5 (1999), no. $1,1-34$.

[23] - Stability analysis in order-preserving systems in the presence of symmetry, Proc. Roy. Soc. Edinburgh Sect. A 129 (1999), no. 2, 395-438. 
[24] E. Risler, Global convergence toward traveling fronts in nonlinear parabolic systems with a gradient structure, Ann. Inst. H. Poincaré Anal. Non Linéaire 25 (2008), no. 2, $381-424$.

[25] V. Roussier, Stability of radially symmetric travelling waves in reaction-diffusion equations, Ann. Inst. H. Poincaré Anal. Non Linéaire 21 (2004), no. 3, 341-379.

[26] Z.-C. Wang, W.-T. Li and S. Ruan, Entire solutions in bistable reaction-diffusion equations with nonlocal delayed nonlinearity, Trans. Amer. Math. Soc. 361 (2009), no. 4, 2047-2084.

[27] J. X. Xin, Multidimensional stability of traveling waves in a bistable reaction-diffusion equation I, Comm. Partial Differential Equations 17 (1992), no. 11-12, 1889-1899.

[28] H. Yagisita, Backward global solutions characterizing annihilation dynamics of travelling fronts, Publ. Res. Inst. Math. Sci. 39 (2003), no. 1, 117-164.

Yang Wang

School of Mathematical Sciences, Shanxi University, Taiyuan, Shanxi, 030006, China E-mail address: ywang2005@sxu.edu.cn

Xiong Li

School of Mathematical Sciences, Beijing Normal University, Beijing, 100875, China E-mail address: xli@bnu.edu.cn 